\title{
EXISTENCE THEORY FOR NONRESONANT SINGULAR BOUNDARY VALUE PROBLEMS
}

\author{
by DONAL O'REGAN
}

(Received 22nd December 1993)

\begin{abstract}
We present some existence results for the "nonresonant" singular boundary value problem $\frac{1}{p q}\left(p y^{\prime}\right)^{\prime}+\mu y=f(t, y)$ a.e. on $[0,1]$ with $\lim _{t \rightarrow 0} p(t) y^{\prime}(t)=y(1)=0$. Here $\mu$ is such that $\frac{1}{p q}\left(p u^{\prime}\right)^{\prime}+\mu u=0$ a.e. on $[0,1]$ with $\lim _{t \rightarrow 0+} p(t) u^{\prime}(t)=u(1)=0$ has only the trivial solution.
\end{abstract}

1991 Mathematics subject classification: 34B15.

\section{Introduction}

This paper establishes existence results for the "nonresonant" singular boundary value problem

$$
\left\{\begin{array}{l}
\frac{1}{p(t) q(t)}\left(p(t) y^{\prime}(t)\right)^{\prime}+\mu y(t)=f(t, y(t)) \quad \text { a.e. on }[0,1] \\
\lim _{t \rightarrow 0^{+}} p(t) y^{\prime}(t)=0 \\
y(1)=0
\end{array}\right.
$$

where $\mu$ is such that

$$
\left\{\begin{array}{l}
\frac{1}{p q}\left(p y^{\prime}\right)^{\prime}+\mu y(t)=0 \quad \text { a.e. on }[0,1] \\
\lim _{t \rightarrow 0+} p(t) y^{\prime}(t)=0 \\
y(1)=0
\end{array}\right.
$$

has only the trivial solution. Throughout the paper $p \in C[0,1] \cap C^{1}(0,1)$ together with $p>0$ on $(0,1)$; also $q$ is measurable with $q>0$ a.e. on $[0,1]$ and $\int_{0}^{1} p(x) q(x) d x<\infty$.

Remarks. (i). Throughout the condition $y(1)=0$ could be replaced by the more general condition $a y(1)+b \lim _{t \rightarrow 1}-p(t) y^{\prime}(t)=0, a>0, b \geqq 0$.

(ii). We do not assume $\int_{0}^{1} \frac{d s}{p(s)}<\infty$.

In addition $f:[0,1] \times \mathbf{R} \rightarrow \mathbf{R}$ will be a Carathéodory function. By this we mean:

(i). $t \rightarrow f(t, y)$ is measurable for all $y \in \mathbf{R}$

(ii). $y \rightarrow f(t, y)$ is a continuous for a.e. $t \in[0,1]$.

For notional purposes let $w$ be a weight function. By $L_{w}^{r}[0,1], r \geqq 1$ we mean the space of functions $u$ such that $\int_{0}^{1} w(t)|u(t)|^{r} d t<\infty$. In particular $L_{w}^{2}[0,1]$ denotes the space of functions $u$ such that $\int_{0}^{1} w(t)|u(t)|^{2} d t<\infty$; also for $u, v \in L_{w}^{2}[0,1]$ define $\langle u, v\rangle=$ 
$\int_{0}^{1} w(t) u(t) \overline{v(t)} d t$. Let $A C[0,1]$ be the space of functions which are absolutely continuous on $[0,1]$.

This paper will be divided into three main sections. Section 2 discusses the linear problem i.e. (1.1) with $f \equiv 0$. In Section 3 fixed point methods (in particular a nonlinear alternative of Leray-Schauder type) is used to obtain an existence principle. The final section establishes some existence results for (1.1); these results extend and complement the theory in $[4,6,21]$.

Finally we remark here that problems of type (1.1) occur in many applications in the physical sciences, for example in radially symmetric nonlinear diffusion $[\mathbf{2 0}, \mathbf{2 2}]$ in the $n$-dimensional sphere we have $p(t)=t^{n-1}$; these problems involve a homogeneous Neumann condition at zero i.e. $\lim _{t \rightarrow 0^{+}} t^{n-1} y^{\prime}(t)=0$. Another important example is the Poisson-Boltzmann equation

$$
\left\{\begin{array}{l}
y^{\prime \prime}+\frac{\alpha}{t} y^{\prime}=f(t, y), 0<t<1 \\
y^{\prime}\left(0^{+}\right)=y(1)=0, \alpha \geqq 1
\end{array}\right.
$$

which occurs in the theory of thermal explosions [3] and in the theory of electrohydrodynamics [11]. The results related to (1.3) in the literature [4] usually consider the situation when inf $\frac{\partial f}{\partial y}$, sup $\frac{\partial f}{\partial y}$ are bounded and satisfy a "nonresonant" condition; here the infimum and supremum are taken over $\{(t, y): 0 \leqq t \leqq 1,-\infty<y<\infty\}$. In this paper we improve the above existence result; in fact in our theory the existence of $\frac{\partial f}{\partial y}$ is not assumed.

\section{Linear problem}

Theorem 2.1. Suppose

$$
\begin{gathered}
p \in C[0,1] \cap C^{1}(0,1) \text { with } p>0 \text { on }(0,1) \\
q \in L_{p}^{1}[0,1] \text { with } q>0 \text { a.e. on }[0,1]
\end{gathered}
$$

and

$$
\int_{0}^{1} \frac{1}{p(s)}\left(\int_{0}^{s} p(x) q(x) d x\right)^{1 / \alpha} d s<\infty \text { for some constant } \alpha>1
$$

are satisfied.

(i) Then

$$
\left\{\begin{array}{l}
\frac{1}{p}\left(p y^{\prime}\right)^{\prime}+\mu q y=0 \quad \text { a.e. on }[0,1] \\
\lim _{t \rightarrow 0+p(t) y^{\prime}(t)=0} \\
y(0)=a_{0} \neq 0
\end{array}\right.
$$

has a solution $y_{1} \in C[0,1] \cap C^{1}(0,1)$ with $p y_{1}^{\prime} \in A C[0,1]$. (By a solution to (2.4) we mean a function $y \in C[0,1] \cap C^{1}(0,1), p y^{\prime} \in A C[0,1]$ which satisfies the differential equation a.e. on $[0,1]$ and the stated conditions.) 
(ii) Then

$$
\left\{\begin{array}{l}
\frac{1}{p}\left(p y^{\prime}\right)^{\prime}+\mu q y=0 \quad \text { a.e. on }[0,1] \\
y(1)=0 \\
\lim _{t \rightarrow 1}-p(t) y^{\prime}(t)=1
\end{array}\right.
$$

has a solution $y_{2} \in L_{p q}^{\alpha}[0,1]$ with $y_{2} \in C(0,1] \cap C^{1}(0,1)$ and $p y_{2}^{\prime} \in A C[0,1]$.

Proof. (i). Let $C[0,1]$ denote the Banach space of continuous functions on $[0,1]$ endowed with the norm

$$
|u|_{K}=\sup _{t \in[0,1]}\left|e^{-K R(t)} u(t)\right| \text { where } R(t)=\int_{0}^{t} p(x) q(x) d x
$$

and

$$
K=\frac{1}{\beta}\left(|\mu| \int_{0}^{1} \frac{1}{p(s)}\left(\int_{0}^{s} p(x) q(x) d x\right)^{1 / \alpha} d s\right)^{\beta}
$$

Remark. Here $\beta=\frac{\alpha}{\alpha-1}$ i.e. $\beta$ and $\alpha$ are conjugate exponents.

Solving (2.4) is equivalent to finding $y \in C[0,1]$ which satisfies

$$
y(t)=a_{0}-\mu \int_{0}^{t} \frac{1}{p(s)} \int_{0}^{s} p(x) q(x) y(x) d x d s .
$$

Define the operator $N: C[0,1] \rightarrow C[0,1]$ by

$$
N y(t)=a_{0}-\mu \int_{0}^{t} \frac{1}{p(s)} \int_{0}^{s} p(x) q(x) y(x) d x d s
$$

Now $N$ is a contraction since

$$
\begin{aligned}
|N u-N v|_{K} & \leqq|\mu||u-v|_{K} \sup _{t \in[0,1]} e^{-K R(t)} \int_{0}^{t} \frac{1}{p(s)} \int_{0}^{s} p(x) q(x) e^{K R(x)} d x d s \\
& \leqq|\mu||u-v|_{K} \sup _{t \in[0,1]} e^{-K R(t)} \int_{0}^{t} \frac{1}{p(s)}\left(\int_{0}^{s} p q d x\right)^{1 / \alpha}\left(\int_{0}^{s} p q e^{\beta K R(x)} d x\right)^{1 / \beta} d s \\
& \leqq|\mu||u-v|_{K} \sup _{t \in[0,1]} e^{-K R(t)} \int_{0}^{t} \frac{1}{p(s)}\left(\int_{0}^{s} p(x) q(x) d x\right)^{1 / \alpha}\left(\frac{e^{\beta K R(s)}}{\beta K}-\frac{1}{\beta K}\right)^{1 / \beta} d s \\
& \leqq \frac{|\mu|}{(\beta K)^{1 / \beta}}|u-v|_{K} \sup _{t \in[0,1]} e^{-K R(t)}\left(e^{1}\{\beta K R(t)\}-1\right)^{1 / \beta} \int_{0}^{t}\left(\int_{0}^{s} p(x) q(x) d x\right)^{1 / \alpha} d s \\
& \leqq\left(1-e^{-\beta K R(1)}\right)^{1 / \beta}|u-v|_{K}
\end{aligned}
$$


using Hölder's integral inequality. The Banach contraction principle now establishes the result.

(ii). Let $L_{p q}^{\alpha}[0,1]$ denote the Banach space of functions $u$, with $\int_{0}^{1} p q|u|^{\alpha} d t<\infty$, endowed with the norm

$$
\|u\|_{K}=\left(\int_{0}^{1} p(t) q(t) e^{-K Q(t)}|u(t)|^{\alpha} d t\right)^{1 / \alpha} \text { where } Q(t)=\int_{t}^{1} p(x) q(x) d x
$$

and

$$
K=\frac{\alpha}{\beta}\left(|\mu|^{\alpha} \int_{0}^{1} p(t) q(t)\left(\int_{t}^{1} \frac{d s}{p(s)}\right)^{\alpha} d t\right)^{\beta / \alpha} \text { where } \beta=\frac{\alpha}{\alpha-1}
$$

Remarks. (i). Notice for example that $\int_{1 / 2}^{1} \frac{d s}{p(s)}<\infty$ since

$$
\int_{1 / 2}^{1} \frac{d s}{p(s)}=\int_{1 / 2}^{1} \frac{\left(\int_{0}^{s} p(x) q(x) d x\right)^{1 / \alpha}}{p(s)\left(\int_{0}^{s} p(x) q(x) d x\right)^{1 / \alpha}} d s \leqq \frac{1}{\left(\int_{0}^{1 / 2} p(x) q(x) d x\right)^{1 / \alpha}} \int_{1 / 2}^{1} \frac{1}{p(s)}\left(\int_{0}^{s} p(x) q(x) d x\right)^{1 / \alpha} d s
$$

(ii). Notice (2.3) implies

$$
\int_{0}^{1} p(t) q(t)\left(\int_{t}^{1} \frac{d s}{p(s)}\right)^{\alpha} d t<\infty
$$

To see this let

$$
g(t)=\left(\int_{t}^{1} \frac{d s}{p(s)}\right)^{\alpha-1}
$$

and fix $\varepsilon, 0<\varepsilon<1$. Interchange the order of integration and use Hölder's inequality to obtain

$$
\begin{aligned}
\int_{\varepsilon}^{1} p(t) q(t) g(t) \int_{t}^{1} \frac{d s}{p(s)} d t & =\int_{\varepsilon}^{1} \frac{1}{p(s)} \int_{\varepsilon}^{s} p(t) q(t) g(t) d t \\
& \leqq\left(\int_{\varepsilon}^{1} p(t) q(t) g^{\beta}(t) d t\right)^{1 / \beta} \int_{\varepsilon}^{1} \frac{1}{p(s)}\left(\int_{\varepsilon}^{s} p(t) q(t) d t\right)^{1 / \alpha} d s .
\end{aligned}
$$

Consequently

$$
\int_{\varepsilon}^{1} p(t) q(t)\left(\int_{1}^{1} \frac{d s}{p(s)}\right)^{\alpha} d t \leqq\left(\int_{\varepsilon}^{1} p(t) q(t)\left(\int_{i}^{1} \frac{d s}{p(s)}\right)^{\alpha} d t\right)^{1 / \beta} \int_{\varepsilon}^{1} \frac{1}{p(s)}\left(\int_{\varepsilon}^{s} p(t) q(t) d t\right)^{1 / \alpha} d s .
$$

We will show that 


$$
y(t)=-\int_{i}^{1} \frac{d s}{p(s)}-\mu \int_{t}^{1} \frac{1}{p(s)} \int_{s}^{1} p(x) q(x) y(x) d x d s
$$

has a solution $y_{2} \in L_{p q}^{\alpha}[0,1]$. Also we will show $y_{2} \in C(0,1] \cap C^{1}(0,1)$ and $p y_{2}^{\prime} \in A C[0,1]$ and consequently $y_{2}$ will be a solution of (2.5).

Define the operator: $L_{p q}^{\alpha}[0,1] \rightarrow L_{p q}^{\alpha}[0,1]$ by

$$
M y(t)=-\int_{1}^{1} \frac{d s}{p(s)}-\mu \int_{i}^{1} \frac{1}{p(s)} \int_{s}^{1} p(x) q(x) y(x) d x d s .
$$

Remark. $\quad M$ is well defined because of $(2.6)$ and

$$
\begin{aligned}
\int_{0}^{1} p q\left(\int_{1}^{1} \frac{1}{p} \int_{s}^{1} p q|y| d x d s\right)^{\alpha} d t & \leqq\left(\int_{0}^{1} p q|y| d x\right)^{a} \int_{0}^{1} p q\left(\int_{1}^{1} \frac{d s}{p(s)}\right)^{\alpha} d t \\
& \leqq\left(\int_{0}^{1} p q|y|^{\alpha} d x\right)\left(\int_{0}^{1} p q d x\right)^{\alpha / \beta} \int_{0}^{1} p q\left(\int_{t}^{1} \frac{d s}{p(s)}\right)^{\alpha} d t
\end{aligned}
$$

for any $y \in L_{p q}^{\alpha}[0,1]$.

Now $M$ is a contraction since

$$
\begin{aligned}
\|M u-M v\|_{K}^{\alpha} & \leqq|\mu|^{\alpha} \int_{0}^{1} p q e^{-K Q(t)}\left(\int_{1}^{1} \frac{1}{p(s)} \int_{s}^{1} p(x) q(x)|u(x)-v(x)| d x d s\right)^{\alpha} d t \\
& \leqq|\mu|^{\alpha} \int_{0}^{1} p q e^{-K Q(t)}\left(\int_{t}^{1} p q e^{-K Q(x) / \alpha} e^{K Q(x) / \alpha}|u(x)-v(x)| d x \int_{t}^{1} \frac{d s}{p(s)}\right)^{\alpha} d t \\
& \leqq|\mu|^{\alpha}\|u-v\|_{K}^{\alpha} \int_{0}^{1} p q e^{-K Q(t)}\left(\int_{t}^{1} p q e^{\beta K Q(x) / \alpha} d x\right)^{\alpha / \beta}\left(\int_{1}^{1} \frac{d s}{p(s)}\right)^{\alpha} d t \\
& \leqq|\mu|^{\alpha}\|u-v\|_{K}^{\alpha} \int_{0}^{1} p q e^{-K Q(t)}\left(\frac{\alpha}{\beta K} e^{\beta K Q(t) / \alpha}-\frac{\alpha}{\beta K}\right)^{\alpha / \beta}\left(\int_{t}^{1} \frac{d s}{p(s)}\right)^{\alpha} d t \\
& \leqq\left(\frac{\alpha}{\beta K}\right)^{\alpha / \beta}|\mu|^{\alpha}\|u-v\|_{K}^{\alpha} \int_{0}^{1} p q\left(1-e^{-\beta K Q(t) / \alpha}\right)^{\alpha / \beta}\left(\int_{1}^{1} \frac{d s}{p(s)}\right)^{\alpha} d t \\
& \leqq\left(1-e^{-\beta K Q(0) / \alpha}\right)^{\alpha / \beta}\|u-v\|_{K}^{\alpha} .
\end{aligned}
$$


The Banach contraction principle now establishes that (2.7) has a solution $y_{2} \in L_{p q}^{\alpha}[0,1]$. Also

$$
p(t) y_{2}^{\prime}(t)=1+\mu \int_{t}^{1} p(x) q(x) y_{2}(x) d x
$$

so $p y_{2}^{\prime} \in A C[0,1]$ since $y_{2} \in L_{p q}^{a}[0,1]$ implies $p q y_{2} \in L^{1}[0,1]$. Thus $y_{2}$ is a solution of $(2.5)$.

\section{Consider now}

$$
\frac{1}{p q}\left(p y^{\prime}\right)^{\prime}+\mu y=h(t) \text { a.e. on }[0,1]
$$

where (2.1), (2.2), (2.3) and

$$
h \in L_{p q}^{\beta}[0,1] ; \text { here } \beta=\frac{\alpha}{\alpha-1}
$$

hold.

Theorem 2.2. Suppose (2.1), (2.2), (2.3) and (2.9) are satisfied. In addition $\mu$ is such that (1.2) has only the trivial solution. Then

$$
\left\{\begin{array}{l}
\frac{1}{p q}\left(p y^{\prime}\right)^{\prime}+\mu y=h(t) \quad \text { a.e. on }[0,1] \\
\lim _{t \rightarrow 0^{+}} p(t) y^{\prime}(t)=0 \\
y(1)=0
\end{array}\right.
$$

has exactly one solution $y$ (note $y \in L_{p q}^{\alpha}[0,1]$ with $y \in C(0,1] \cap C^{1}(0,1)$ and $p y^{\prime} \in A C[0,1]$ ) given by

$$
y(t)=\int_{0}^{1} G(t, s) q(s) h(s) d s
$$

where $G(t, s)$ is the Green's function i.e.

$$
G(t, s)=\left\{\begin{array}{l}
\frac{y_{1}(s) y_{2}(t)}{W(s)}=c_{0} p(s) y_{1}(s) y_{2}(t), 0<s \leqq t \\
\frac{y_{1}(t) y_{2}(s)}{W(s)}=c_{0} p(s) y_{2}(s) y_{1}(t), 0 \leqq s<1
\end{array}\right.
$$

Here $y_{1}$ and $y_{2}$ are as described in Theorem 2.1 and $W(s)$ is the Wronksian of $y_{1}$ and $y_{2}$ at $s$ and notice $p(s) W(s)=\left(1 / c_{0}\right) \neq 0$ for $s \in[0,1]$. 
Proof. This follows the standard construction of the Green's function; see [22, 24] for example. We will just justify that $p(s) W(s) \neq 0$ for $s \in[0,1]$. To see this all one needs to show is that $y_{1}(1) \neq 0$. If $y_{1}(1)=0$ then $y_{1}$ satisfies (1.2) and consequently $y_{1} \equiv 0$. This contradicts the fact that $y(0)=a_{0} \neq 0$.

Remark. Notice $y$ in (2.11) is in $L_{p q}^{\alpha}[0,1]$ since

$$
\int_{0}^{1} p(t) q(t)\left(\int_{t}^{1} p(s) q\left|y_{2}(s) y_{1}(t) h(s)\right| d s\right)^{\alpha} d t \leqq \int_{0}^{1} p q\left|y_{1}\right|^{\alpha}\left(\int_{t}^{1} p q\left|y_{2}\right|^{\alpha} d s\right)\left(\int_{t}^{1} p q|h|^{\beta} d s\right)^{\alpha / \beta} d s
$$

and so

$$
\int_{0}^{1} p(t) q(t)\left(\int_{0}^{t} p(s) q(s)\left|y_{1}(s) y_{2}(t) h(s)\right| d s\right)^{\alpha} d t<\infty
$$

\section{Existence principle}

We use a nonlinear alternative of Leray-Schauder type [9] to establish our existence principle. By a map being compact we mean it is continuous with relatively compact range. A map is completely continuous if it is continuous and the image of every bounded set in the domain is contained in a compact set in the range.

Theorem 3.1. Assume $U$ is a relatively open subset of a convex set $K$ in a Banach space $E$. Let $N: \bar{U} \rightarrow K$ be a compact map with $0 \in U$. Then either

(i) $N$ has a fixed point in $\bar{U}$; or

(ii) there is a $u \in \partial U$ and $a \lambda \in(0,1)$ such that $u=\lambda N u$.

Next we gather some well known results [12] from the theory of nonlinear integral equations.

Theorem 3.2. Let $\alpha>1$ be a constant and $f:[0,1] \times \mathbf{R} \rightarrow \mathbf{R}$ be a Carathéodory function. Define the operator

$$
F y(t)=f(t, y(t))
$$

and suppose $F: L_{p q}^{\alpha}[0,1] \rightarrow L_{p q}^{\beta}[0,1]$; here $\beta=\frac{\alpha}{\alpha-1}$. Then $F$ is continuous and bounded.

Theorem 3.3. Consider the linear integral operator

$$
A y(t)=\int_{0}^{1} p(s) q(s) k(t, s) y(s) d s
$$

with 


$$
\int_{0}^{1} p(t) q(t) \int_{0}^{1} p(s) q(s)|k(t, s)|^{\alpha} d s d t<\infty \text { for some } \alpha>1 \text {. }
$$

Then $A: L_{p q}^{\beta}[0,1] \rightarrow L_{p q}^{\alpha}[0,1], \beta=\frac{\alpha}{\alpha-1}$ is completely continuous.

We next prove an existence principle for (1.1).

Theorem 3.4. Let $f:[0,1] \times \mathbf{R} \rightarrow \mathbf{R}$ be a Carethéodory function and suppose (2.1), (2.2) and (2.3) are satisfied. Also suppose

$$
f(t, y(t)) \in L_{p q}^{\beta}[0,1] \quad \text { whenever } y \in L_{p q}^{\alpha}[0,1] ; \text { here } \beta=\frac{\alpha}{\alpha-1} \text {. }
$$

In addition $\mu$ is such that (1.2) has only the trivial solution. Now suppose there is a constant $M_{0}$, independent of $\lambda$, with

$$
\|y\|=\left(\int_{0}^{1} p(t) q(t)|y(t)|^{\alpha} d t\right)^{1 / \alpha} \leqq M_{0}
$$

for any solution $y$ (here $y \in L_{p q}^{\alpha}[0,1]$ with $y \in C(0,1] \cap C^{1}(0,1)$ and $\left.p y^{\prime} \in A C[0,1]\right)$ to

$$
\left\{\begin{array}{l}
\frac{1}{p q}\left(p y^{\prime}\right)^{\prime}+\mu y=\lambda f(t, y) \quad \text { a.e. on }[0,1] \\
\lim _{t \rightarrow 0+} p(t) y^{\prime}(t)=0 \\
y(1)=0
\end{array}\right.
$$

for each $\lambda \in(0,1)$. Then $(1.1)$ has at least one solution.

Proof. Solving (3.3) $)_{\lambda}$ is equivalent to finding $y \in L_{p q}^{\alpha}[0,1]$ which satisfies

$$
y(t)=\lambda \int_{0}^{1} p(s) q(s) k(t, s) f(s, y(s)) d s
$$

where

$$
k(t, s)=\left\{\begin{array}{l}
c_{0} y_{1}(s) y_{2}(t), 0<s \leqq t \\
c_{0} y_{2}(s) y_{1}(t), t \leqq s<1
\end{array}\right.
$$

and $y_{1}, y_{2}, c_{0}$ are described in Theorem 2.2. Define the operator $N: L_{p q}^{\alpha}[0,1] \rightarrow L_{p q}^{\alpha}[0,1]$ by

$$
N y(t)=\int_{0}^{1} p(s) q(s) k(t, s) f(s, y(s) d s
$$


Remark. $\quad N$ is well defined since

$$
\int_{0}^{1} p q\left(\int_{t}^{1} p q\left|y_{1}(t) y_{2}(s) f(s, y)\right| d s\right)^{\alpha} d t \leqq \int_{0}^{1} p q\left|y_{1}\right|^{\alpha}\left(\int_{t}^{1} p q\left|y_{2}\right|^{\alpha} d s\right)\left(\int_{t}^{1} p q|f(s, y)|^{\beta} d s\right)^{\alpha / \beta} d t
$$

and so

$$
\int_{0}^{1} p(t) q(t)\left(\int_{0}^{t} p(s) q(s)\left|y_{1}(s) y_{2}(t) f(s, y(s))\right| d s\right)^{\alpha} d t<\infty
$$

Next define $F: L_{p q}^{\alpha}[0,1] \rightarrow L_{p q}^{\beta}[0,1]$ by

$$
F y(t)=f(t, y(t))
$$

and $A: L_{p q}^{\beta}[0,1] \rightarrow L_{p q}^{\alpha}[0,1]$ by

$$
A y(t)=\int_{0}^{1} p(s) q(s) k(t, s) y(s) d s .
$$

Notice (3.2) and Theorem 3.2 implies $F$ is bounded and continuous. $A$ is completely continuous by Theorem 3.3 .

Remark. Notice $\int_{0}^{1} p(t) q(t) \int_{0}^{1} p(s) q(s)|k(t, s)|^{\alpha} d s d t<\infty$ since

$$
\int_{0}^{1} p(t) q(t)\left(\int_{0}^{t} p(s) q(s)\left|y_{1}(s) y_{2}(t)\right|^{\alpha} d s d t \leqq \int_{0}^{1} p(t) q(t)\left|y_{2}(t)\right|^{\alpha} \int_{0}^{1} p(s) q(s)\left|y_{1}(s)\right|^{\alpha} d s d t<\infty\right.
$$

and so

$$
\int_{0}^{1} p(t) q(t)\left(\int_{1}^{1} p(s) q(s)\left|y_{2}(s) y_{1}(t)\right|^{\alpha} d s d t<\infty\right.
$$

Consequently $N=A F: L_{p q}^{\alpha}[0,1] \rightarrow L_{p q}^{\alpha}[0,1]$ is completely continuous. Set

$$
U=\left\{u \in L_{p q}^{\alpha}[0,1]:\|u\|<M_{0}+1\right\}, K=E=L_{p q}^{\alpha}[0,1] .
$$

Then Theorem 3.1 implies that $N$ has a fixed point i.e. (1.1) has a solution $y \in L_{p q}^{\alpha}[0,1]$. The fact that $y \in C(0,1] \cap C^{1}(0,1)$ with $p y^{\prime} \in A C[0,1]$ follows from (3.4) with $\lambda=1$.

\section{Existence theory}

Theorem 4.1. Let $f:[0,1] \times \mathbf{R} \rightarrow \mathbf{R}$ be a Carathéodory function and suppose (2.1), (2.2) 
and (2.3) are satisfied. In addition $\mu$ is such that (1.2) has only the trivial solution. Let $\beta=\frac{\alpha}{\alpha-1}$. Now assume

$$
\left\{\begin{array}{l}
|f(t, u)| \leqq \phi_{1}(t)+\phi_{2}(t) \psi(|u|) \text { a.e. on }[0,1] \text { where } \phi_{1}^{\beta}, \phi_{2} \in L_{p q}^{1}[0,1] \\
\text { and } \psi:[0, \infty) \rightarrow[0, \infty) \text { is a continuous function }
\end{array}\right.
$$

$$
\left\{\begin{array}{l}
\text { there exists } Q_{0} \geqq 0 \text { and a continuous function } \theta:[0, \infty) \rightarrow[0, \infty) \text { with } \\
\int_{0}^{1} p(s) q(s) \phi_{2}^{\beta}(s) \psi^{\beta}(|y(s)|) d s \leqq Q_{0} \theta(\|y\|) \text { for any } y \in L_{p q}^{\alpha}[0,1] \\
\text { here }\|y\|=\left(\int_{0}^{1} p(t) q(t)|y(t)|^{\alpha} d t\right)^{1 / \alpha}
\end{array}\right.
$$

and

$$
\left\{\begin{array}{l}
A_{0} \equiv 2^{q 0} c_{0}^{\alpha} Q_{0}^{\alpha / \beta}\left\|y_{2}\right\|^{\alpha}\left\|y_{1}\right\|^{\alpha} \limsup _{x \rightarrow \infty} \frac{(\theta(x))^{\alpha / \beta}}{x^{\alpha}}<1 \text { where } y_{1}, y_{2}, c_{0} \\
\text { are as described in Theorem } 2.2 \text { and } q_{0}=\frac{2 \alpha^{2} \beta-\beta^{2}-\alpha^{2}+\alpha \beta}{\alpha \beta}
\end{array}\right.
$$

are satisfied. Then (1.1) has at least one solution.

Remarks. (i). Notice (3.2) is automatically satisfied since (4.2) holds and also since $\phi_{1}^{\beta} \in L_{p q}^{1}[0,1]$.

(ii). If $\psi(|u|)=|u|^{\gamma}, 0 \leqq \gamma<\min \left\{\frac{\alpha}{\beta}, 1\right\}$ and $\phi_{2}^{\beta \alpha /(\alpha-\beta \gamma)} \in L_{p q}^{1}[0,1]$ then (4.2) and (4.3) are satisfied since

$$
\int_{0}^{1} p(s) q(s) \phi_{2}^{\beta}(s)|y(s)|^{\beta \gamma} d s \leqq\|y\|^{\beta \gamma}\left(\int_{0}^{1} p q \phi_{2}^{\beta \alpha /(\alpha-\beta \gamma)} d s\right)^{(\alpha-\beta \gamma) / \alpha} \text { for any } y \in L_{p q}^{\alpha}[0,1]
$$

and so with $\theta(x)=x^{\beta \gamma}$ we have

$$
\limsup _{x \rightarrow \infty} \frac{(\theta(x))^{\alpha / \beta}}{x^{\alpha}}=\limsup _{x \rightarrow \infty} x^{\alpha(\gamma-1)}=0 .
$$

Proof. Let $y$ be a solution to $(3.3)_{\lambda}$ for $0<\lambda<1$. Then

$$
y(t)=\lambda c_{0} y_{2}(t) \int_{0}^{1} p(s) q(s) y_{1}(s) f(s, y(s)) d s+\lambda c_{0} y_{1}(t) \int_{t}^{1} p(s) q(s) f(s, y(s)) d s
$$

where $y_{1}, y_{2}, c_{0}$ are as described in Theorem 2.2. Recall $\left(a_{0}+b_{0}\right)^{r_{0}} \leqq 2^{r_{0}-1}\left(a_{0}^{r_{0}}+b_{0}^{r_{0}}\right), a_{0} \geqq$ $0, b_{0} \geqq 0, r_{0} \geqq 1$ so

$$
\begin{aligned}
\|y\|^{\alpha} & \leqq 2^{\alpha-1} c_{0}^{\alpha} \int_{0}^{1} p(t) q(t)\left|y_{2}(t)\right|^{\alpha}\left(\int_{0}^{t} p(s) q(s)\left|y_{1}(s) \| f(s, y(s))\right| d s\right)^{\alpha} d t \\
& +2^{\alpha-1} c_{0}^{\alpha} \int_{0}^{1} p(t) q(t)\left|y_{1}(t)\right|^{\alpha}\left(\int_{t}^{1} p(s) q(s)\left|y_{2}(s) \| f(s, y(s))\right| d s\right)^{\alpha} d t
\end{aligned}
$$


This together with Hölder's inequality implies

$$
\|y\|^{\alpha} \leqq 2^{\alpha} c_{0}^{\alpha}\left\|y_{2}\right\|^{\alpha}\left\|y_{1}\right\|^{\alpha}\left(\int_{0}^{1} p(s) q(s)|f(s, y(s))|^{\beta} d s\right)^{\alpha / \beta} .
$$

In addition

$$
\begin{aligned}
\int_{0}^{1} p(s) q(s)|f(s,(s))|^{\beta} d s & \leqq 2^{\beta-1} \int_{0}^{1} p(s) q(s) \phi_{1}^{\beta}(s) d s+2^{\beta-1} \int_{0}^{1} p(s) q(s) \phi_{2}^{\beta}(s) \psi^{\beta}(|y(s)|) d s \\
& \leqq 2^{\beta-1} \int_{0}^{1} p(s) q(s) \phi_{1}^{\beta}(s) d s+2^{\beta-1} Q_{0} \theta(\|y\|) .
\end{aligned}
$$

This inequality together with $\left(a_{0}+b_{0}\right)^{1 / r_{0}} \leqq 2^{\left(r_{0}-1\right) / r_{0}}\left(a_{0}^{1 / r_{0}}+b_{0}^{1 / r_{0}}\right), a_{0} \geqq 0, b_{0} \geqq 0, r_{0} \geqq 1$ or $\left(a_{0}+b_{0}\right)^{s_{0}} \leqq 2^{s_{0}-1}\left(a_{0}^{s_{0}}+b_{0}^{s_{0}}\right), s_{0} \geqq 1$ and (4.4) implies

$$
\|y\|^{\alpha} \leqq 2^{\alpha} c_{0}^{\alpha}\left\|y_{2}\right\|^{\alpha}\left\|y_{1}\right\|^{\alpha} 2^{(\alpha-\beta) / \alpha}\left(2^{\alpha(\beta-1) / \beta}\left(\int_{0}^{1} p q \phi_{1}^{\beta} d s\right)^{\alpha / \beta}+2^{\alpha(\beta-1) / \beta} Q_{0}^{\alpha / \beta}(\theta(\|y\|))^{\alpha / \beta}\right) .
$$

Consequently

$$
1 \leqq 2^{q_{0}} c_{0}^{\alpha}\left\|y_{2}\right\|^{\alpha}\left\|y_{1}\right\|^{\alpha}\left(\frac{\left(\int_{0}^{1} p q \phi_{1}^{\beta} d s\right)^{\alpha / \beta}}{\|y\|^{\alpha}}+\frac{Q_{0}^{\alpha / \beta}(\theta(\|y\|))^{\alpha / \beta}}{\|y\|^{\alpha}}\right)
$$

Thus there exists a constant $M_{0}$, independent of $\lambda$, with $\|y\| \leqq M_{0}$ for any solution $y$ satisfying (3.3) $)_{\lambda}$ i.e. $y=\lambda N y$ where $N$ is as described in Theorem 3.4. If this was not true then there exists $u_{n}=\lambda_{n} N u_{n}$ with $\left\|u_{n}\right\| \rightarrow \infty$ as $n \rightarrow \infty$ and since $\lim \sup \left(s_{n}+t_{n}\right) \leqq$ $\lim \sup s_{n}+\lim \sup t_{n}$ for any sequences $s_{n} \geqq 0, t_{n} \geqq 0$ we have from (4.6) that $1 \leqq A_{0}$, a contradiction (see (4.3)). Thus there exists a constant $M_{0}$, independent of $\lambda$, with $\|y\| \leqq M_{0}$ and the result now follows from Theorem 3.4.

The next two existence results extend in a "particular direction" Theorem 4.1 if certain criteria are fulfilled. To discuss the first result we begin by gathering together some facts on the singular eigenvalue problem

$$
\left\{\begin{array}{l}
L u=\lambda u \text { a.e. on }[0,1] \\
\lim _{t \rightarrow 0^{+}} p(t) u^{\prime}(t)=0 \\
u(1)=0
\end{array}\right.
$$

where $L u=-\frac{1}{p q}\left(p u^{\prime}\right)^{\prime}$. Assume (2.1), (2.2) and

$$
\int_{0}^{1} \frac{1}{p(s)}\left(\int_{0}^{s} p(x) q(x) d x\right)^{1 / 2} d s<\infty
$$


hold.

Remarks. (i). In this case $\alpha=2$ in (2.3).

(ii). Here $t=0$ is a singular point in the limit circle case $[18,19,24]$.

Let

$$
\begin{gathered}
D(L)=\left\{\omega \in C[0,1]: w, p w^{\prime} \in A C[0,1] \text { with } \frac{1}{p q}\left(p w^{\prime}\right)^{\prime} \in L_{p q}^{2}[0,1]\right. \\
\text { and } \left.\lim _{t \rightarrow 0^{+}} p(t) w^{\prime}(t)=w(1)=0\right\}
\end{gathered}
$$

In $[18,19]$ it was shown that $L^{-1}: L_{p q}^{2}[0,1] \rightarrow D(L)$ and $L^{-1}$ is completely continuous with $\left\langle L^{-1} u, v\right\rangle=\left\langle u, L^{-1} v\right\rangle$ for $u, v \in L_{p q}^{2}[0,1]$. Consequently the spectral theorem for compact self adjoint operators [24] implies that $L$ has a countably infinite number of real eigenvalues $\lambda_{i}$ with corresponding eigenfunctions $\psi_{i} \in D(L)$. The eigenfunctions $\psi_{i}$ may be chosen so that they form an orthonormal set and we may arrange the eigenvalues so that

$$
\lambda_{0}<\lambda_{1}<\lambda_{2}<\ldots
$$

The following Rayleigh-Ritz minimization theorem $[18,19]$ also holds.

Theorem 4.2. Suppose (2.1), (2.2) and (4.8) hold. Then

$$
\lambda_{0} \int_{0}^{1} p(t) q(t) y^{2}(t) d t \leqq \int_{0}^{1} p(t)\left[y^{\prime}(t)\right]^{2} d t
$$

for all functions $y \in D(L)$.

We can improve the result in Theorem 4.1 if (4.8) holds and if $\mu<\lambda_{0}$; here $\lambda_{0}$ is the first eigenvalue of (4.7). In particular consider

$$
\left\{\begin{array}{l}
\frac{1}{p q}\left(p y^{\prime}\right)^{\prime}=f(t, y) \text { a.e. on }[0,1] \\
\lim _{t \rightarrow 0^{+}} p(t) y^{\prime}(t)=0 \\
y(1)=0
\end{array}\right.
$$

Theorem 4.3. Let $f:[0,1] \times \mathbf{R} \rightarrow \mathbf{R}$ be a Carathéodory function and suppose (2.1), (2.2) and (4.8) are satisfied. Also assume 


$$
f(t, y(t)) \in L_{p q}^{2}[0,1] \text { whenever } y \in L_{p q}^{2}[0,1] .
$$

In addition suppose $f(t, u)=g(t, u)+h(t, u)$ with $g, h:[0,1] \times \mathbf{R} \rightarrow \mathbf{R}$ Carathéodory functions and

$$
\begin{gathered}
\left\{\begin{array}{l}
|u h(t, u)| \leqq \phi_{1}(t)|u|+\phi_{2}(t) \rho(|u|) \text { a.e. on }[0,1] \text { where } \rho:[0, \infty) \rightarrow[0, \infty) \\
\text { is a nondecreasing continuous function }
\end{array}\right. \\
\qquad u g(t, u) \geqq-\mu_{0} u^{2} \text { for a.e. } t \in[0,1] \text { and } u \in \mathbf{R} ; \text { here } \mu_{0}<\lambda_{0} \\
\int_{0}^{1} p(t) q(t) \phi_{1}(t)\left(\int_{1}^{1} \frac{d s}{p(s)}\right)^{1 / 2} d t<\infty \text { and } \int_{0}^{1} p(t) q(t) \phi_{2}(t) \rho\left(\left(\int_{t}^{1} \frac{d s}{p(s)}\right)^{1 / 2}\right) d t<\infty \\
\left\{\begin{array}{l}
\text { there exist constants } Q_{1}\left(\text { independent of } a_{0} \text { and } b_{0}\right) \text { and } Q_{2} \text { such that for any } \\
a_{0} \geqq 0, b_{0} \geqq 0 \text { we have } \rho\left(a_{0} b_{0}\right) \leqq Q_{1} \rho\left(a_{0}\right) \rho\left(b_{0}\right)+Q_{2} \rho\left(b_{0}\right)
\end{array}\right.
\end{gathered}
$$

and

$$
\left\{\begin{array}{l}
A_{1} \equiv Q_{1}\left(\int_{0}^{1} p(t) q(t) \phi_{2}(t) \rho\left(\left(\int_{t}^{1} \frac{d s}{p(s)}\right)^{1 / 2}\right) d t\right) \limsup \frac{\rho(x)}{x^{2}}<\eta_{0} \\
\text { with } \eta_{0}=1 \text { if } \mu_{0}<0 \text { whereas } \eta_{0}=1-\frac{\mu_{0}}{\lambda_{0}} \text { if } 0 \leqq \mu_{0}<\lambda_{0}
\end{array}\right.
$$

are satisfied. Then (4.9) has at least one solution.

Remark. If $\rho(|u|)=|u|^{\gamma+1}, 0 \leqq \gamma<1$ and $\int_{0}^{1} p(t) q(t) \phi_{2}(t)\left(\int_{t}^{1} \frac{d s}{p(s)}\right)^{(\gamma+1) / 2} d t<\infty$ then (4.13), (4.14) and (4.15) are satisfied since if $Q_{1}=1, Q_{2}=0$ we have $\rho\left(a_{0} b_{0}\right)=\left|a_{0} b_{0}\right|^{p+1}=$ $\left|a_{0}\right|^{p+1}\left|b_{0}\right|^{\gamma+1}$ and also

$$
\limsup _{x \rightarrow \infty} \frac{\rho(x)}{x^{2}}=\limsup _{x \rightarrow \infty} x^{\nu-1}=0
$$

Proof. Let $y$ be a solution to

$$
\left\{\begin{array}{l}
\frac{1}{p q}\left(p y^{\prime}\right)^{\prime}=\lambda f(t, y) \text { a.e. on }[0,1] \\
\lim _{t \rightarrow 0^{+}} p(t) y^{\prime}(t)=0 \\
y(1)=0
\end{array}\right.
$$

for $0<\lambda<1$. Multiply the differential equation by $-y$ and integrate from 0 to 1 to obtain 


$$
\begin{aligned}
\left\|y^{\prime}\right\|_{0}^{2} & \leqq-\lambda \int_{0}^{1} p q y g(t, y) d t+\int_{0}^{1} p q|y h(t, y)| d t \\
& \leqq \lambda \mu_{0}\|y\|^{2}+\int_{0}^{1} p q\left[\phi_{1}(t)|y(t)|+\phi_{1}(t) \rho(|y(t)|)\right] d t
\end{aligned}
$$

where for notational purposes $\|u\|^{2}=\int_{0}^{1} p q|u|^{2} d t$ and $\|u\|_{0}^{2}=\int_{0}^{1} p|u|^{2} d t$. Apply Theorem 4.2 if $0 \leqq \mu_{0}<\lambda_{0}$ to obtain

$$
\eta_{0}\left\|y^{\prime}\right\|_{0}^{2} \leqq \int_{0}^{1} p q \phi_{1}|y| d t+\int_{0}^{1} p q \phi_{2} \rho(|y(t)|) d t
$$

where $\eta_{0}$ is as described in (4.15). Also for $t \in(0,1)$ we have from Hölder's inequality that

$$
|y(t)| \leqq\left\|y^{\prime}\right\|_{0}\left(\int_{t}^{1} \frac{d s}{p(s)}\right)^{1 / 2}
$$

and this together with (4.17) and the fact that $\rho$ is nondecreasing yields

$$
\eta_{0}\left\|y^{\prime}\right\|_{0}^{2} \leqq N_{1}\left\|y^{\prime}\right\|_{0}+\int_{0}^{1} p(t) q(t) \phi_{2}(t) \rho\left(\left\|y^{\prime}\right\|_{0}\left(\int_{t}^{1} \frac{d s}{p(s)}\right)^{1 / 2}\right) d t
$$

where $N_{1}=\int_{0}^{1} p q \phi_{1}\left(\int_{t}^{1} \frac{d s}{p(s)}\right)^{1 / 2} d t$. Using (4.14) we obtain

$$
\eta_{0}\left\|y^{\prime}\right\|_{0}^{2} \leqq N_{1}\left\|y^{\prime}\right\|_{0}+Q_{1} N_{2} \rho\left(\left\|y^{\prime}\right\|_{0}\right)+Q_{2} N_{2}
$$

where $N_{2}=\int_{0}^{1} p q \phi_{2} \rho\left(\left(\int_{t}^{1} \frac{d s}{p(s)}\right)^{1 / 2}\right) d t$. Consequently

$$
\eta_{0} \leqq \frac{N_{1}\left\|y^{\prime}\right\|_{0}+Q_{2} N_{2}}{\left\|y^{\prime}\right\|_{0}^{2}}+\frac{Q_{1} N_{2} \rho\left(\left\|y^{\prime}\right\|_{0}\right)}{\left\|y^{\prime}\right\|_{0}^{2}}
$$

Thus (as in Theorem 4.1) exists a constant $M_{1}$, independent of $\lambda$, with $\left\|y^{\prime}\right\|_{0} \leqq M_{1}$ for any solution $y$ to $(4.16)_{\lambda}$. This together with Theorem 4.2 yields

$$
\int_{0}^{1} p q|y|^{2} d t \leqq \frac{1}{\lambda_{0}} M_{1}^{2}
$$

so the result follows from Theorem 3.4 (with $\mu=0$ and $\alpha=2$ ).

Finally we examine the boundary value problem (4.9) where in the case $p q f:[0,1] \times$ $\mathbf{R} \rightarrow \mathbf{R}$ is an $L^{1}$-Carathéodory function. By this we mean:

(i) $p q f:[0,1] \times \mathbf{R} \rightarrow \mathbf{R}$ is a Carathéodory function, and 
(ii) for any $r>0$ there exists $h_{r} \in L^{1}[0,1]$ with $|p(t) q(t) f(t, u)| \leqq h_{r}(t)$ for a.e. $t \in[0,1]$ and for all $|u| \leqq r$.

For the remainder of the paper assume (2.1), (2.2) and

$$
\int_{0}^{1} \frac{1}{p(s)} \int_{0}^{s} p(x) q(x) d x d s<\infty
$$

and

$$
\int_{0}^{1} \frac{1}{p(s)} \int_{0}^{s} p(x) q(x) h_{r}(x) d x d s<\infty \text { for any } r>0 \text {; here } h_{r} \text { is as described above }
$$

hold. In $[8,18]$ we proved the following existence principle.

Theorem 4.4. Let pqf: $[0,1] \times \mathbf{R} \rightarrow \mathbf{R}$ be a $L^{1}$-Carathéodory function with (2.1), (2.2), (4.19) and (4.20) holding. In addition suppose there is a constant $M_{0}$, independent of $\lambda$, with

$$
|y|_{0}=\sup _{[0,1]}|y(t)| \leqq M_{0}
$$

for any solution $y$ (here $y \in C[0,1] \cap C^{1}(0,1)$ with $\left.p y^{\prime} \in A C[0,1]\right)$ to

$$
\left\{\begin{array}{l}
\frac{1}{p q}\left(p y^{\prime}\right)^{\prime}=\lambda f(t, y) \text { a.e. on }[0,1] \\
\lim _{t \rightarrow 0^{+}} p(t) y^{\prime}(t)=0 \\
y(1)=0
\end{array}\right.
$$

for each $0<\lambda<1$. Then (4.9) has at least one solution.

Theorem 4.5. Let $p q f:[0,1] \times \mathbf{R} \rightarrow \mathbf{R}$ be a $L^{1}$-Carathéodory function with (2.1), (2.2) and (4.19) holding. In addition suppose

$$
\begin{gathered}
\left\{\begin{array}{l}
|f(t, u)| \leqq \phi_{1}(t)+\phi_{2}(t) \Omega(|u|) \text { a.e. on }[0,1] \text { where } \Omega:[0, \infty) \rightarrow[0, \infty) \\
\text { is a nondecreasing continuous function }
\end{array}\right. \\
\qquad \int_{0}^{1} \frac{1}{p(s)} \int_{0}^{s} p(x) q(x) \phi_{i}(x) d x d s<\infty, i=1,2
\end{gathered}
$$

and

$$
\left(\int_{0}^{1} \frac{1}{p(s)} \int_{0}^{s} p(x) q(x) \phi_{2}(x) d x d s\right) \limsup _{x \rightarrow \infty} \frac{\Omega(x)}{x}<1
$$


are satisfied. Then (4.9) has at least one solution.

Proof. Let $y$ be a solution to $(4.21)_{\lambda}$ for $0<\lambda<1$. Then for $t \in[0,1]$ we have

$$
y(t)=-\int_{t}^{1} \frac{1}{p(s)} \int_{0}^{s} p(x) q(x) f(x, y(x)) d x d s
$$

and so

$$
|y(x)| \leqq \int_{i}^{1} \frac{1}{p(s)} \int_{0}^{s} p(x) q(x) \phi_{1}(x) d x d s+\int_{1}^{1} \frac{1}{p(s)} \int_{0}^{s} p(x) q(x) \phi_{2}(x) \Omega(|y(x)|) d x d s .
$$

Now $|y(x)| \leqq \sup _{[0,1]}|y(s)| \equiv|y|_{0}$ and this together with the fact that $\Omega$ is nondecreasing yields

$$
|y(t)| \leqq \int_{0}^{1} \frac{1}{p(s)} \int_{0}^{s} p(x) q(x) \phi_{1}(x) d x d s+\Omega\left(|y|_{0}\right) \int_{0}^{1} \frac{1}{p(s)} \int_{0}^{s} p(x) q(x) \phi_{2}(x) d x d s .
$$

Let $K_{i}=\int_{0}^{1} \frac{1}{p(s)} \int_{0}^{s} p(x) q(x) \phi_{i}(x) d x d s, i=1,2$ so

$$
|y|_{0} \leqq K_{1}+K_{2} \Omega\left(|y|_{0}\right)
$$

and consequently

$$
1 \leqq \frac{K_{1}}{|y|_{0}}+\frac{K_{2} \Omega\left(|y|_{0}\right)}{|y|_{0}}
$$

Thus (as in Theorem 4.1) there exists a constant $M_{0}$, independent of $\lambda$, with $|y|_{0} \leqq M_{0}$ for any solution $y$ to $(4.21)_{\lambda}$ The result follows from Theorem 4.4.

\section{REFERENCES}

1. F. V. Atkinson, Discrete and continuous boundary problems (Academic Press, New York, 1964).

2. L. E. Bobisud and D. O'ReGan, Positive solutions for a class of nonlinear singular boundary value problems at resonance, J. Math. Anal. Appl 184 (1994), 263-284.

3. P. L. Chambre, On the solution of the Poisson-Boltzmann equation with application to the theory of thermal explosions, J. Chem. Phys. 20 (1952), 1795-1797.

4. M. M. Chawla and P. N. Shivakumar, On the existence of solutions of a class of singular nonlinear two point boundary value problems, J. Comput. Appl. Math. 19 (1987), 379-388.

5. D. R. Dunninger and J. C. Kurtz, A priori bounds and existence of positive solutions for singular nonlinear boundary value problems, SIAM J. Math. Anal. 17 (1986), 595-609.

6. M. A. El-Gebeily, A. Boumenir and A. B. M. Elgind, Existence and uniqueness of solutions 
of a class of two-point singular nonlinear boundary value problems, J. Comput. Appl. Math., 46 (1993), 345-355.

7. A. FondA and J. MAWHIN, Quadratic forms, weighted eigenfunctions and boundary value problems for nonlinear second order differential equations, Proc. Royal Soc. Edinburgh 112A (1989), 145-153.

8. M. Frigon and D. O'Regan, Some general existence principles for ordinary differential equations, Topological Methods in Nonlinear Anal. 2 (1993), 35-54.

9. A. Granas, R. B. Guenther and J. W. Lee, Some general existence principles in the Carathéodory theory of nonlinear differential systems, J. Math. Pures Appl. 70 (1991), 153-196.

10. R. Iannacci and M. N. Nkashama, Nonlinear two-point boundary value problems at resonance without Landesman-Lazer condtions, Proc. Amer. Math. Soc. 106 (1989), 943-952.

11. J. B. Keller, Electrodynamics $I$. The equilibrium of a charged gas in a container, $J$. Rat. Mech. Anal. 5 (1957), 715-724.

12. M. A. KRASNOSELSKII, Topological methods in the theory of nonlinear integral equations (MacMillan Co., New York, 1964).

13. J. Mawhin, J. R. Ward and M. Willem, Necessary and sufficient conditions for the solvability of a nonlinear two point boundary value problem, Proc. Amer. Math. Soc. 93 (1985), 667-674.

14. J. Mawhin and W. Omano, Two point boundary value problems for nonlinear perturbations of some singular linear differential equations at resonance, Comment. Math. Univ. Carolinae 30 (1989), 537-550.

15. J. W. Mooney, Numerical schemes for degenerate boundary value problems, J. Phys. A. 26 (1993), 413-421.

16. M. A. Najmark, Linear differential operators Part II (Ungar Publ. Co., London, 1968).

17. D. O'Regan, Solvability of some two point boundary value problems of Dirichlet, Neumann or Periodic type, Dynamic Systems and Appl. 2 (1993), 163-182.

18. D. O'ReGan, Singular Sturm Liouville problems and existence of solutions to singular nonlinear boundary value problems, Nonlinear Anal. 20 (1993), 767-779.

19. D. O'Regan, Existence theory for singular two point boundary value problems, in Proc. Fourth Int. Coll. Diff. Eq. (Plovdiv, Bulgaria, Int. Science Publ., Utrecht, 1994), 215-228.

20. D. O'REGAN, Existence principles for second order nonresonant boundary value problems, J. Appl. Math. Stoch. Anal. 7 (1994), 487-507.

21. D. O'ReGaN, Nonresonant and resonant singular boundary value problems, to appear.

22. D. Powers, Boundary value problems (Harcourt Brace Jovanovich, San Diego, 1987).

23. L. SANCHEZ, Positive solutions for a class of semilinear two point boundary value problems, Bull. Austral. Math. Soc. 45 (1992), 439-451.

24. I. Stakgold, Greens functions and boundary value problems (John Wiley and Sons, New York, 1979).

Department of Mathematics

University College Galway

GaLWAY

IRELAND 